\title{
Turning loss into gain
}

\author{
The exploitation of non-Hermitian quantum physics concepts in classical photonics has spawned much research \\ activity during 2017. We conclude the year with a focus issue on parity-time symmetry and concepts with \\ overlapping goals, in particular topological and non-reciprocal photonics.
}

W hen many of us in photonics think of propagating light through materials with a negative imaginary part of the dielectric permittivity, that is, dissipative media, we instinctively cringe at the prospect of dreaded optical losses. Loss is typically viewed as something to avoid by any means, such as by using alternative materials, or designing the system to minimize the overlap of the photons with the lossy region. It is this scenario that has trained many of us to think of a positive imaginary part of the dielectric permittivity, or gain, primarily as a means to compensate for losses, or even to overcome them to achieve net gain. However, a different way of thinking of the role of both gain and loss has emerged in photonics over recent years as it turns out that a system exhibiting an appropriate balancing act between the two can enable entirely new capabilities thanks to the concept of parity-time (PT) symmetry photonics.

The roots of the PT-symmetry concept lie in mathematical and quantum physics where we deal with Hamiltonian problems in which real-world observables such as energy and momentum result from Hermitian operators, since their eigenvalues are real. However, it turns out that complex, or non-Hermitian, operators can also yield real eigenvalues. And while this non-Hermiticity may be a difficulty in the quantum realm, it is not a problem in the field of classical photonics; complex eigenstates with gain and loss are commonplace in optics. Balancing them in the manner required for non-Hermitian photonics devices requires planning, but it is possible and may not be much of a deterrent when considering what may be gained.

In a Q\&A on page 742 of this issue, Demetrios Christodoulides explains that setting up a system for PT-symmetry photonics is neither as difficult, nor as fragile, as one might imagine. In fact, Christodoulides highlights the robustness of the phenomena. But now that we know that these quantum physics concepts translate into classical photonics, why should we be excited? Well, as Christodoulides points out, the concepts actually enable new capabilities that we did not have access to before. One outcome discussed is the potential for fundamentally more sensitive detectors due to dimensional collapse that can occur in such systems.

For a general overview and introduction to non-Hermitian photonics, see page 752, where Liang Feng, Ramy El-Ganainy and $\mathrm{Li} \mathrm{Ge}$ review the topic. They also explain that creating and superposing non-Hermitian eigenstates is relatively easy in photonics with gain and loss, whereas in quantum electronics experimental demonstrations remain elusive.

While one might think that PT-symmetry photonics has just emerged, a Google Scholar search for "parity-time symmetry" + "laser" yields $\sim 5,700$ results, dating from 2016 to the present. Even the sub-field of 'exceptional points' - points that mark a transition to symmetry breaking and splitting of eigensolutions in the complex plane - has $\sim 4,000$ results (also dating from 2016) when searched together with "non-Hermitian photonics" or "nonHermitian optics".

One of the most active areas in the field of PT symmetry is related to findings showing that the idea can yield topologically protected modes, even in the continuum without a bandgap, thanks to exceptional points. Topological photonics in general shares many of the goals of PT symmetry and is arguably reshaping the way we think about propagation and scattering. Waveguides that only allow propagation in one direction, reflectionfree sharp waveguide bends, robust delay lines and truly non-reciprocal devices are all possible. There are a number of different concepts and approaches that can be exploited to achieve topologically protected photonic states; see page 763 for a review of the latest advances in topological photonics by Alexander Khanikaev and Gennady Shvets. Their Review highlights that there has been much recent experimental progress.

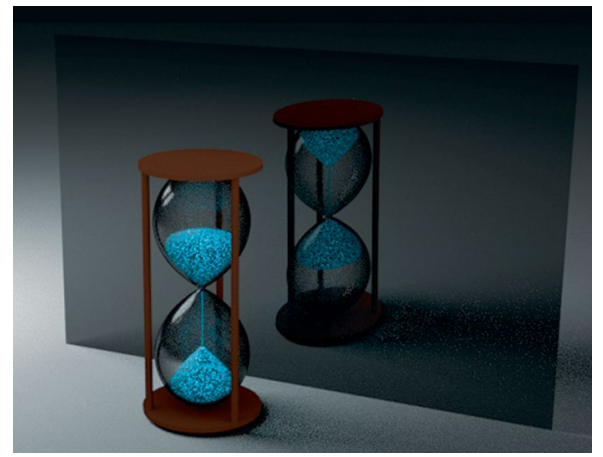

Credit: Jessica Brassard, Michigan Technological University

There are now many ways to achieve some form of symmetry breaking or non-reciprocity in photonics, with a major goal being one-way optical devices. A particularly active direction is non-reciprocal systems realized without traditional magnetic materials by employing dynamic modulation of optical or structural parameters. If the challenges can be overcome, such approaches may be more amenable to use in small size integrated systems than bulky magnetic set-ups. On page 774, Dimitrios Sounas and Andrea Alù review the proposals and development of the time modulation approach to non-reciprocal photonics.

Investigation of these new topics appears to have gained momentum over the past year, with various optics communities paying attention, from traditional laser communities (particularly with regard to PT symmetry) to those more focused on nanophotonics or metamaterials. Although there are real challenges ahead for PT symmetry, topological photonics and new approaches to non-reciprocal devices in general, photonics has clearly expanded its toolbox and we look forward to seeing what the confluence of these communities brings over the coming years, and hope that authors will consider Nature Photonics as an outlet for their exciting findings.

Published online: 30 November 2017 https://doi.org/10.1038/s41566-017-0067-2 